\title{
Double-peaked [O I] Profile: A Likely Signature of the Gaseous Ring around KH 15D
}

\author{
Min Fang ${ }^{1,2}$ (1) , Ilaria Pascucci ${ }^{2,3}$ (1) , Jinyoung Serena Kim ${ }^{1,2}$ (1) , and Suzan Edwards ${ }^{4}$ \\ ${ }^{1}$ Department of Astronomy, University of Arizona, 933 North Cherry Avenue, Tucson, AZ 85721, USA \\ ${ }^{2}$ Earths in Other Solar Systems Team, NASA Nexus for Exoplanet System Science, USA \\ ${ }^{3}$ Department of Planetary Sciences, University of Arizona, 1629 East University Boulevard, Tucson, AZ 85721, USA \\ ${ }^{4}$ Five College Astronomy Department, Smith College, Northampton, MA 01063, USA \\ Received 2019 March 11; revised 2019 June 6; accepted 2019 June 11; published 2019 July 1
}

\begin{abstract}
$\mathrm{KH} 15 \mathrm{D}$ is a well-known spectroscopic binary because of its unique and dramatic photometric variability. The variability is explained by a circumbinary dust ring, but the ring itself was never directly detected. We present a new interpretation of the double-peaked [O I] $\lambda 6300$ profiles as originating from the hot disk surface of KH $15 \mathrm{D}$. By modeling these profiles, we measure emitting radii between $\sim 0.5$ and 5 au, basically a gaseous ring very similar in radial extent to the dust ring inferred from modeling the system's photometric variability. We also discuss the possibility that external photoevaporation driven by ultraviolet photons from the nearby massive star HD 47887 has truncated the outer edge of the disk to the observed value.
\end{abstract}

Key words: open clusters and associations: individual (NGC 2264) - protoplanetary disks - stars: individual (KH 15D) - stars: pre-main sequence

\section{Introduction}

$\mathrm{KH} 15 \mathrm{D}$ is a pre-main sequence (PMS) spectroscopic binary $(\mathrm{A}+\mathrm{B})$ with near-equal-mass components $\left(0.715 M_{\odot}\right.$ for Star A and $0.74 M_{\odot}$ for Star B), high eccentricity $(e \approx 0.6)$, a semimajor axis of $\sim 0.25 \mathrm{au}$, and a period of 48.37 days (Johnson et al. 2004; Winn et al. 2006; Aronow et al. 2018). It is a member of the NGC 2264 cluster at $\sim 750 \mathrm{pc}$, based on the members from Venuti et al. 2018 and Gaia Data Release 2 parallaxes, and the cluster is about 2-6 Myr old (Naylor 2009; Lim et al. 2016). KH 15D exhibits unique and dramatic longand short-term photometric variability (e.g., Capelo et al. 2012). From 2006 to 2009 Star B was fully occulted, while after 2010 Star A became occulted; currently, the entirety of Star B is visible at each apastron passage (Aronow et al. 2018) The short-term variability has the same period as the binary (Kearns \& Herbst 1998; Hamilton et al. 2001; Herbst et al. 2002; Johnson et al. 2004). However, the large depth (several magnitudes) and long duration (days to tens of days) of the eclipse, in combination with the time variability, cannot be solely attributed to the binary. Additional occultation by circumbinary matter must be invoked (Hamilton et al. 2001; Herbst et al. 2002).

A nearly edge-on, warped, and precessing circumbinary ring of dust $(\sim 1$ to $5 \mathrm{au})$ is thought to occult the eccentric PMS binary and explain the available light curves (Chiang \& Murray-Clay 2004; Winn et al. 2004). However, the circumbinary disk eluded detection. The system shows no infrared excess emission out to $8 \mu \mathrm{m}$ (Arulanantham et al. 2016), in line with the relatively large inner dust edge. In addition, the dust ring has not been detected at millimeter wavelengths with the Submillimeter Array (SMA) or the Atacama Large Millimeter/ submillimeter Array (ALMA). The millimeter observations have yielded upper limits on the total (gas+dust) circumbinary disk mass of $1.7 M_{\text {JUP }}$ (Aronow et al. 2018), just a factor of $\sim 2$ lower than the mean protoplanetary disk mass in young (1-3 Myr) star-forming regions (e.g., Ansdell et al. 2016; Pascucci et al. 2016). Interestingly, the SMA CO $J=3-2$ line reveals a bipolar collimated outflow, the northern lobe of which is spatially coincident with the $\mathrm{H}_{2}$ jet associated with $\mathrm{KH} 15 \mathrm{D}$
(Tokunaga et al. 2004; Aronow et al. 2018). Finally, the system presents broad $\mathrm{H} \alpha$ line profiles, indicative of active accretion (Hamilton et al. 2012), perhaps funneled from gas surrounding both stars (see the case of the spectroscopic binary DQ Tau; Muzerolle et al. 2019). The presence of a jet and ongoing accretion point to the presence of a gaseous disk.

Here, we interpret the double-peaked [O I] $\lambda 6300$ profiles from $\mathrm{KH} 15 \mathrm{D}$ as originating from the surface of this gaseous disk (Section 2). Modeling of the line profiles reveals that the emitting gas is radially confined (Section 3). We discuss how external photoevaporation could have truncated the gaseous disk to the outer edge inferred from our [O I] $\lambda 6300$ modeling (Section 4).

\section{Double-peaked [O I] $\lambda 6300$ Profiles}

The spectroscopic data presented in this work were obtained from the Very Large Telescope (VLT)/Ultraviolet and Visual Echelle Spectrograph (UVES), European Southern Observatory (ESO) archive for three nights in 2001 and six nights in 2004 with a spectral resolution of $\sim 42,000$ and have been already published in Hamilton et al. $(2003,2012)$ and Mundt et al. (2010). We extracted the raw data from the ESO archive ${ }^{5}$ and reduced them with the ESO UVES pipelines under the ESO Recipe Flexible Execution Workbench environment (Freudling et al. 2013). We obtain 21 spectra that cover both [O I] $\lambda 6300$ and $\mathrm{H} \alpha$ lines. The 2001 December 14 and 21 and the 2004 December 16-18 (UT) spectra were taken during Star A eclipse phase, the spectrum acquired on 2001 December 29 was out of eclipse, and the spectra obtained on 2004 December 13-15 were approaching Star A eclipse phase (Mundt et al. 2010; Hamilton et al. 2012).

We removed telluric absorption and subtracted photospheric features near the [O I] $\lambda 6300$ and $\mathrm{H} \alpha$ lines using a K7 PMS template (see e.g., Fang et al. 2018 for details). We adopted a heliocentric radial velocity $v_{\text {helio }}=18.676 \mathrm{~km} \mathrm{~s}^{-1}$ as the the systemic velocity (Mundt et al. 2010; Hamilton et al. 2012).

\footnotetext{
5 Based on observations collected at the European Southern Observatory under ESO programmes 267.C-5736(A) and 074.C-0604(A).
} 


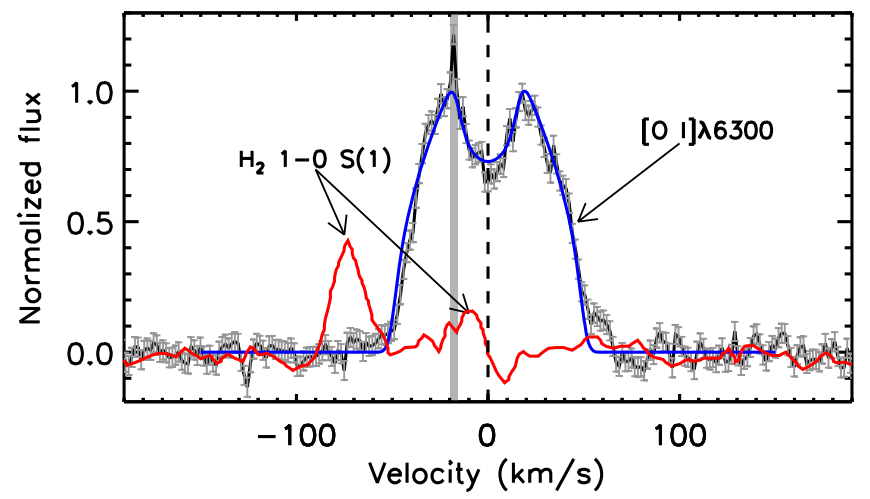

Figure 1. $[\mathrm{O} \mathrm{I}] \lambda 6300$ line profile (gray line) in the system's reference frame obtained on 2001 December 21. The profile is compared with the $\mathrm{H}_{2} 1-0 \mathrm{~S}(1)$ digitally extracted from Figure 2 of Deming et al. (2004; red line). The best-fit model profile is also shown in the panel (blue line). The gray filled region marks the wavelength range contaminated by the sky line and thus excluded in the fitting (see the discussion in Section 2).

This value is consistent with the error-weighted mean radial velocity $\left(19.4 \pm 0.1 \mathrm{~km} \mathrm{~s}^{-1}\right)$ of other NGC 2264 cluster members (Kounkel et al. 2016). We shifted the [O I] $\lambda 6300$ lines to the systemic velocity and present the spectrum with the highest signal-to-noise ratio $(\mathrm{S} / \mathrm{N})$, the one obtained on 2001 December 21, in Figure 1. Individual $\mathrm{H} \alpha$ and [O I] $\lambda 6300$ profiles are shown in Figures 2 and 5.

The [O I] $\lambda 6300$ emission shows the same double-peaked profile at all observing epochs. Note that the sharp blueshifted spikes in some profiles are due to sky contamination because: (1) they are truly spikes, i.e., their width is much narrower than the spectral resolution $\left(\sim 7 \mathrm{~km} \mathrm{~s}^{-1}\right)$; (2) sky lines peak near these spikes and, as they get stronger, so do the spikes; (3) for four spectra taken in 2001 covering also the [O I] $\lambda 5577$ line, we detect the same strong and sharp spikes we see in the [O I] $\lambda 6300$ profiles. The background $\mathrm{H} \alpha$ line, produced mostly by the $\mathrm{HII}$ region (Cone Nebula) around $\mathrm{KH} \mathrm{15D}$, is also present but the ratio of its intensity to the source emission is much smaller than the sky lines at the $[\mathrm{O}$ I] $\lambda 6300$ emission. This explains why we do not see such spikes in the reduced $\mathrm{H} \alpha$ profiles (see Figure 2).

The [O I] $\lambda 6300$ profiles for KH15D differ from those found in most accreting T Tauri stars (TTSs). In TTSs with higher accretion luminosity, the $[\mathrm{O} \mathrm{I}] \lambda 6300$ profiles typically show both a high-velocity component (usually but not exclusively blueshifted) attributed to a jet and a low-velocity component (LVC), which is also often blueshifted (Hartigan et al. 1995). The LVC is found in a broader range of TTSs, including those with low accretion luminosity and transition disks, and itself can consist of both a broad and a narrow component, which have been attributed to slow disk winds from the inner or outer disk, respectively (Hartigan et al. 1995; Simon et al. 2016; Fang et al. 2018; Banzatti et al. 2019). In some cases, the LVC is centered on the stellar velocity, and may be bound material on the surface of the disk; see, e.g., Figure 24 in Simon et al. (2016). The LVCs are always single peaked and have line widths that correlate with disk inclination, indicative of broadening by Keplerian rotation.

The [O I] $\lambda 6300$ profile in $\mathrm{KH} 15 \mathrm{D}$ is distinctive from other TTSs. The profile shows symmetric red and blue peaks ${ }^{6}$ at

\footnotetext{
6 We have mirrored and the subtracted the original spectrum in Figure 1 and find no asymmetry greater than three times the rms next to the line.
}

radial velocities of $\pm 20 \mathrm{~km} \mathrm{~s}^{-1}$ and looks similar over multiple epochs. ${ }^{7}$ While such low velocities are characteristic of TTS LVCs, no other LVCs to date have shown a double-peaked structure. A jet close to the plane of the sky can have low radial velocities, as can be inferred for some TTSs where line ratios for several forbidden lines reveal properties characteristic of shocked, rather than thermally excited, gas for a few sources that would be classified as LVC based on their kinematic properties (Fang et al. 2018). This is the interpretation proposed by Mundt et al. (2010), that the [O I] $\lambda 6300$ profile from $\mathrm{KH} 15 \mathrm{D}$ arises in a pair of identical approaching and receding jets in the plane of the sky. However, in this interpretation we would expect to see variable profiles at different epochs as noticed in other TTSs (Simon et al. 2016). Therefore, we put forward an alternate possibility, informed by recent work on TTS forbidden lines, that the [O I] $\lambda 6300$ traces the surface of a disk and is broadened by Keplerian rotation. Its double-peaked structure, unlike other LVCs in TTSs, would result from the fact that the disk is more like a ring, rather than extended beyond $\sim 5 \mathrm{au}$, as required to fill in the central dip in most TTSs (Hartigan et al. 1995; Simon et al. 2016).

\section{H $\alpha$ Profiles}

An extensive analysis of $\mathrm{H} \alpha$ profiles and associated emission variability is provided in Hamilton et al. (2012). The analyzed spectra covered five contiguous observing seasons (from 2001 to 2006) over which Star A was fully visible as well as partially occulted (in ingress and egress events) and fully occulted. Some of these spectra are the same from which we have extracted the [O I] $\lambda 6300$ profiles and are presented in Figure 2 in order to emphasize the difference in the line profiles of $\mathrm{H} \alpha$ and $[\mathrm{O} \mathrm{I}] \lambda 6300$.

The out-of-eclipse and ingress spectra (left panel) are characterized by an inverse P-Cygni-type profile with a strong redshifted absorption whose changes in centroid velocity can be explained by an accretion stream onto A (Hamilton et al. 2012). The eclipse and egress spectra (right panel) present a more symmetric profile with often more pronounced broad extended wings (up to several hundreds $\mathrm{km} \mathrm{s}^{-1}$ ), which are characteristic of actively accreting stars. Additional spectra in this phase can be seen in Figure 9 of Hamilton et al. (2012). Mundt et al. (2010) interpreted the eclipse profiles as tracing the same bipolar jet as [O I] $\lambda 6300$ because $\mathrm{H} \alpha$ also shows red and blue peaks, although the blue/red intensity is quite variable and the velocities are somewhat higher than [O I] $\lambda 6300$. Alternatively, we might still be seeing accretion onto A (and perhaps $\mathrm{A}+\mathrm{B}$ ) through reflected light from the back of the wall of the circumbinary disk. This could explain the similarity of some ingress and mid-eclipse $\mathrm{H} \alpha$ profiles and has been proposed by Hamilton et al. (2012) to explain the presence of the broad wings during these phases.

While a detailed analysis of the $\mathrm{H} \alpha$ profiles is beyond the scope of this work, we draw the reader's attention to the difference between the $\mathrm{H} \alpha$ and [O I] $\lambda 6300$ profiles. While the

\footnotetext{
7 Note that the claimed factor of $\sim 2$ decrease in the [O I] $\lambda 6300$ flux on 2001 December 20 (Mundt et al. 2010; Hamilton et al. 2012) is likely spurious. The ESO archive does not contain any spectrum acquired on that date. Instead, most likely, the correct spectrum is the one obtained on December 21st; the line profile is the same as in Mundt et al. (2010). Because KH 15D brightened by $0.77 \mathrm{mag}$ in the $R$-band from 2001 December 20 to 21 (Windemuth \& Herbst 2014) and this magnitude is used to convert the line equivalent width into flux, an incorrect assignment of the $R$-band can explain the factor of $\sim 2$ underestimation in the $[\mathrm{O} \mathrm{I}] \lambda 6300$ flux.
} 

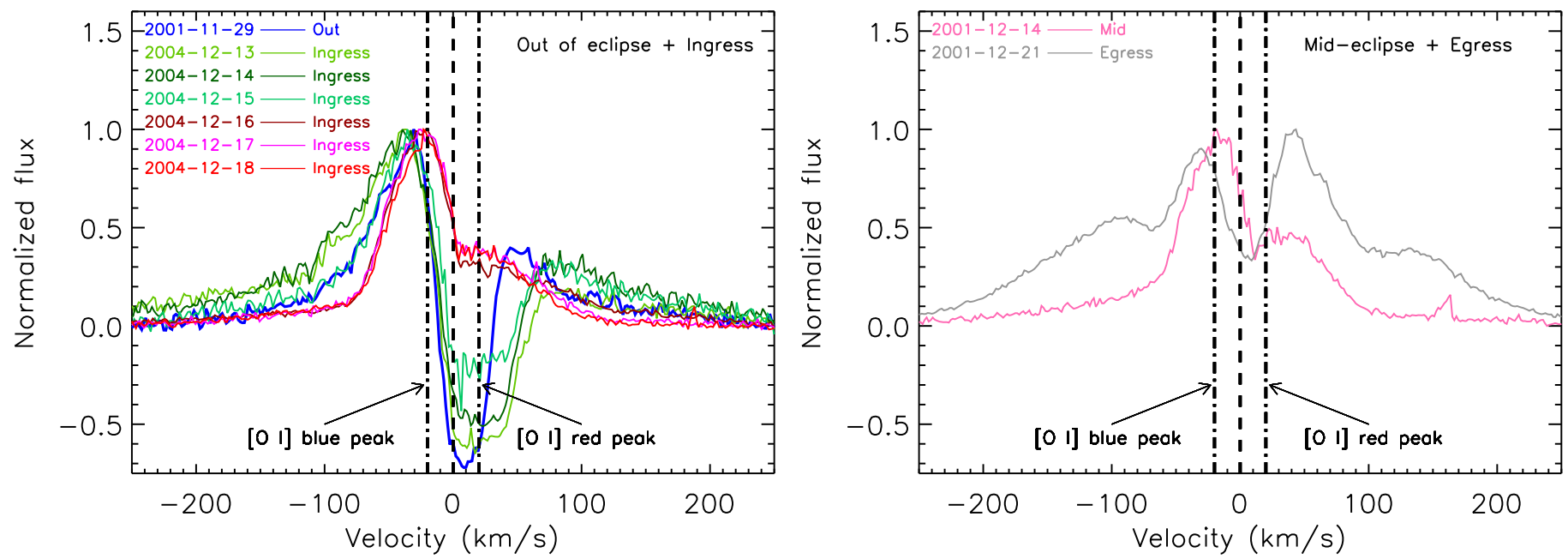

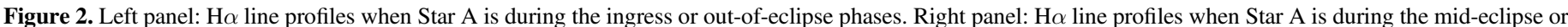

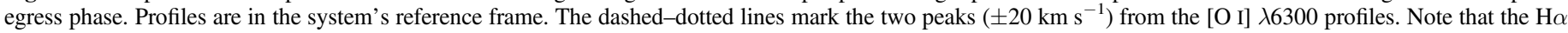
profiles shown here and [O I] $\lambda 6300$ profiles in Figure 5 are extracted from the same spectra, hence correspond to the same observing times.

$\mathrm{H} \alpha$ profiles, even during eclipse, are mostly asymmetric and variable in flux, the [O I] $\lambda 6300$ profiles are persistently more symmetric through all phases (Figure 5) and show only minor variations in flux (Figure 1 in Mundt et al. 2010). This is different from the $[\mathrm{OI}] \lambda 6300$ emission tracing jets, which show strongly variable line intensities and profiles as the emission arises in time variable knots of shocked gas (e.g., Hartigan et al. 2011, Simon et al. 2016). Therefore, our preferred explanation is that the two lines trace different phenomena, with the $[\mathrm{O} I] \lambda 6300$ being more stable, probing the surface of the gaseous circumbinary disk.

\section{A Circumbinary Gaseous Ring}

Here, we explore the possibility that the [O I] $\lambda 6300$ emission originates from the surface of a circumbinary gaseous disk and use the line profile to constrain its size. In the assumption of Keplerian broadening, the $\sim 40 \mathrm{~km} \mathrm{~s}^{-1}$ separation of the two peaks points to a characteristic [O I] emitting radius of $\sim 3 \mathrm{au}$, which is consistent with the mean circumstellar dust ring radius inferred from modeling the photometric variability of KH 15D (Chiang \& Murray-Clay 2004).

To model in more depth the entire line profile, we adopt a power-law distribution for the $\mathrm{O}$ I surface density: $\Sigma(\mathrm{O}$ I $) \propto$ $r^{\alpha}$, where $r$ is the radial distance from the star. We assume optically thin emission and an edge-on $\left(i=90^{\circ}\right)$ disk for simplicity, because (1) photometric variability points to such high disk inclination (Herbst et al. 2002), and (2) the [O I] $\lambda 6300$ line profile is nearly symmetric with two peaks (see Figure 1). The line modeling includes thermal broadening for a temperature of $5000 \mathrm{~K}$ (Simon et al. 2016) and convolution with the instrumental width of $\sim 7 \mathrm{~km} \mathrm{~s}^{-1}$. We vary $\alpha$ from -4 to 4 , the inner disk radius $\left(r_{\text {in }}\right)$ from 0.05 to 2.55 au and outer radius $\left(r_{\text {out }}\right)$ from $r_{\text {in }}$ out to 13 au to perform a grid search and find the parameters leading to the lowest reduced $\chi^{2}$. We focus the fit on the [O I] $\lambda 6300$ line profile obtained during the night of 2001 December 21 because of its high S/N. We further normalize the observed line profile to its peak and fit it with modeled line profiles that are similarly normalized to their peaks. From this grid search we find the following best-fit parameters: $\alpha=-2.9, r_{\text {in }}=0.57 \mathrm{au}$, and $r_{\text {out }}=5.2 \mathrm{au}$. We also implement a Markov Chain Monte Carlo (MCMC) procedure (Sharma 2017) to find the best-fit parameters and evaluate their uncertainties. The posterior distributions with the best-fit parameters and their uncertainties are shown in Figure 3. These values are consistent with those obtained through the grid search. Note that this best-fit profile to the 2001 December 21 spectrum reproduces rather well all other profiles (see Figures 1 and 5), again highlighting the temporal stability of the [O I] $\lambda 6300$ emission.

It is also worth noting that the [O I] $\lambda 6300$ surface brightness distribution follows a rather steep power law: $I \approx r^{-2.8}$, in comparison to other disks (Hartigan et al. 1995; Simon et al. 2016). As pointed out in Hartigan et al. (1995), a steep powerlaw index of -3 is expected if the emission is proportional to the flux from the central star and the disk is flat. Thus, our modeling results further support a disk origin. Interestingly, the inner and outer gas disk radii inferred from the $[\mathrm{O} \mathrm{I}]$ emission are very close to those of the dust ring derived from light curve modeling (see also the next Section). These results together point to a disk with a relatively large inner radius and a very small outer radius, a circumbinary ring of gas.

Meijerink et al. (2012) investigated the effect of high-energy stellar photons on the disk structure and the gas chemical composition by varying the $\mathrm{X}$-ray radiation from 0 to $10^{32} \mathrm{erg} \mathrm{s}^{-1}$ and the far-ultraviolet (FUV) from $10^{29}$ to $10^{32} \mathrm{erg} \mathrm{s}^{-1}$. Their calculations show that there exists a radially extended hot $(\sim 5000 \mathrm{~K})$ region on top of the disk surface $^{8}$ with hydrogen densities of $\sim 10^{6}-10^{8} \mathrm{~cm}^{-3}$. The [O I] $\lambda 6300$ line should be tracing this region.

Mundt et al. (2010) claimed that there is a slight blue asymmetry (14\%) in the [O I] $\lambda 6300$ line profiles. For a system like the KH 15D with a close binary, the [O I] emitting regions can be disturbed by the time varying potential of the binary system (Thun et al. 2017), causing some variability and asymmetry in the [O I] $\lambda 6300$ line profile as proposed by Mundt et al. (2010). However, as discussed in Section 2, the blue part of [O I] $\lambda 6300$ line profile is also contaminated by sky lines, hence changes restricted to a narrow range of velocities close to the sky lines should be interpreted with caution.

\footnotetext{
8 From $z / r \sim 0.2$ at $r \sim 0.6$ au to $z / r \sim 0.5$ at $r \sim 5$ au, where $z$ is the disk vertical height and $r$ is the radial distance from the star.
} 


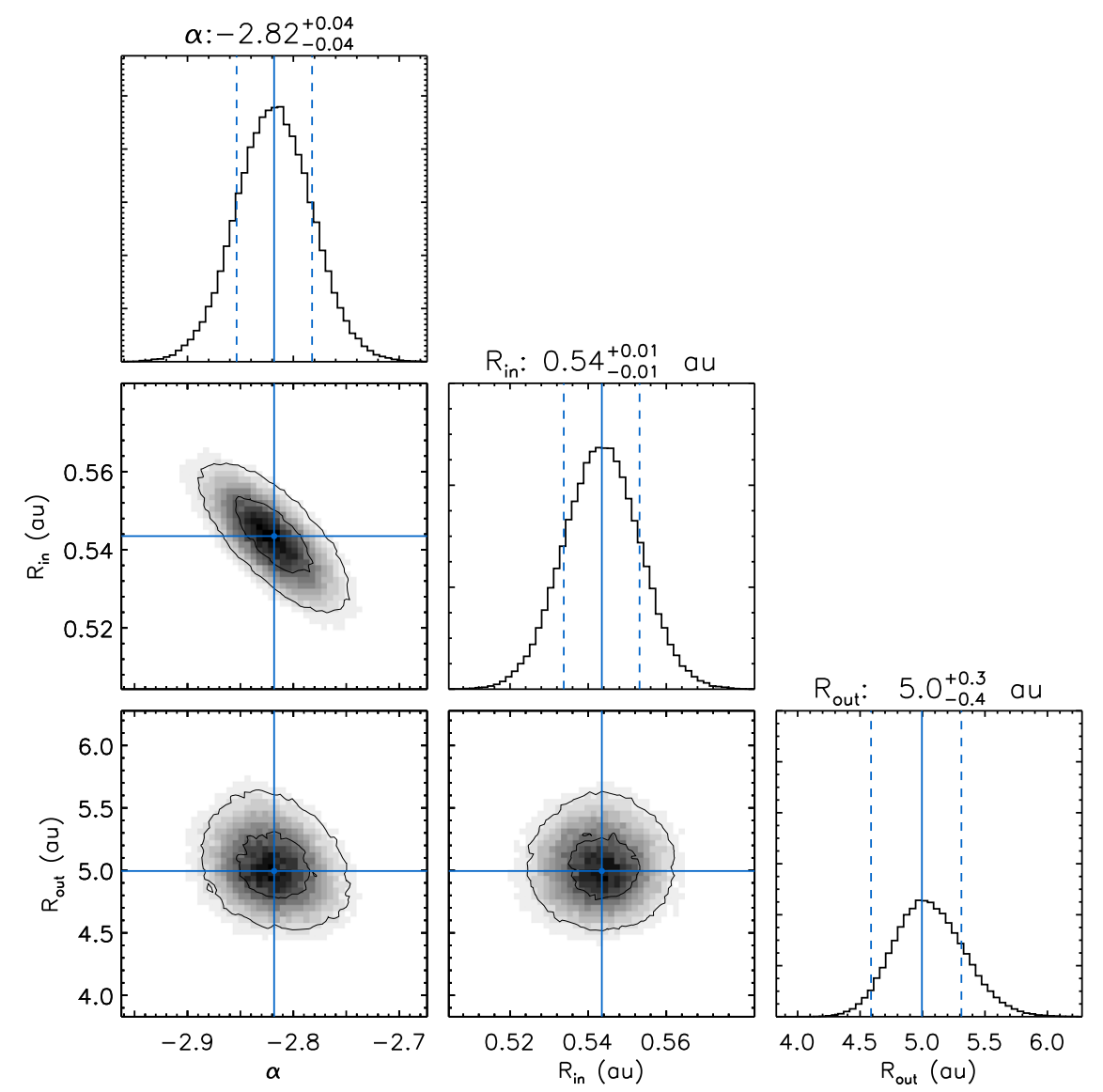

Figure 3. Corner plot showing the posterior distributions from the MCMC fit of the [O I] $\lambda 6300$ line obtained on 2001 December 21 (see Figure 1). The vertical dashed lines are the 16 and 85 percentiles, respectively. The solid lines indicate the medians of the posterior distributions.

Excluding these, likely spurious, features we do not identify any asymmetry in the highest $\mathrm{S} / \mathrm{N}[\mathrm{O} \mathrm{I}] \lambda 6300$ profile.

\section{Inner and Outer Truncation of the Disk}

Several theoretical papers have shown that the inner region of a disk surrounding a close binary is quickly dissipated (e.g., Lin \& Papaloizou 1993; Artymowicz \& Lubow 1994). Recently, Pichardo et al. (2008) carried out numerous simulations, using the test particle approah, to derive a simple relation between the gap radius and binary semimajor axis $(a)$, eccentricity $(e)$, and primary/companion mass ratios $(q)$ : $R_{\text {gap }} \approx 1.93 a\left(1+1.01 e^{0.32}\right)[q(1-q)]^{0.043}$. For the KH $15 \mathrm{D}$ system, $a \sim 0.25$ au, $e \sim 0.6$, and $q \sim 0.83-0.97$ (Winn et al. 2006; Aronow et al. 2018), thus the disk inner radius is expected to be at $\sim 0.8$ au according to these simulations. However, as shown in the more detailed physical models of Muñoz \& Lai (2016) and Muñoz et al. (2019), it is actually difficult to define the cavity radius for an eccentric binary, as gas from the circumbinary disk flows in generating complex and transit streams. Therefore, we consider our estimate of $\sim 0.5$ au from modeling the [O I] $\lambda 6300$ line to be pretty close to the possible cavity radius.

Chiang \& Murray-Clay (2004) argued that the disk of $\mathrm{KH} 15 \mathrm{D}$ cannot extend beyond $\sim 5$ au to maintain rigid precession and explain the long-term photometric variability of the system (but see Lodato \& Facchini 2013 for the possibility of the disk extending to 5-10 au). Recently, Arulanantham et al. (2016) confirmed the rigid precession of the ring and estimated a velocity (across the sky) of $\sim 15 \mathrm{~m} \mathrm{~s}^{-1}$ for the projected edge of the ring. Then, the precession rate of the ascending node of the ring can be estimated to be $0^{\circ} .18 \mathrm{yr}^{-1} / \bar{a}$, where $\bar{a}$ is the mean radius of the circumbinary ring in astronomical units. Using the approximate expression relating the precession rate of ascending node of an inclined circumbinary ring to the mean ring radius from Chiang \& Murray-Clay (2004), the masses (0.715 and $0.74 M_{\odot}$; Aronow et al. 2018), and the orbit $(0.25 \mathrm{au}$; Winn et al.

2004) of the binary, the mean radius of the ring should be $\sim 4$ au. This value is also close to the characteristic [O I] $\lambda 6300$ emitting radius, see Section 4.

Preventing the outer boundary from viscous spreading may require some sort of outside confinement. Chiang \& MurrayClay (2004) proposed a planet exterior to the ring. Here, we explore external photoevaporation as an alternative. At a projected separation of $\sim 37^{\prime \prime}$, southwest from KH $15 \mathrm{D}$, there is a massive star HD $47887\left(T_{\text {eff }}=24,000 \pm 1000 \mathrm{~K}\right.$, Fossati et al. 2014). Based on Gaia Data Release 2, KH 15D and HD 47887 have similar parallaxes $(1.2691 \pm 0.0751$ mas versus $1.3272 \pm 0.0751 \mathrm{mas})$. Thus, it is very likely that both of them belong to the NGC 2264 cluster. With a distance from us of $\sim 750 \mathrm{pc}$, the projected distance between them is $\sim 0.135 \mathrm{pc}$.

Taking this projected distance, the model atmosphere from Lanz \& Hubeny (2007), and the broadband photometry from Kharchenko (2001), the UV field strength near KH 15D produced by HD 47887 is $\sim 4470 G_{0}{ }^{9}{ }^{\text {Under such UV field }}$

\footnotetext{
9 G0 is the Habing unit of UV radiation corresponding to the integrated flux $\left(1.6 \times 10^{-3} \mathrm{erg}^{-1} \mathrm{~cm}^{-2} \mathrm{~s}^{-1}\right)$ over the wavelength range $912 \AA$ to $2400 \AA$ used in Haworth et al. (2018).
} 


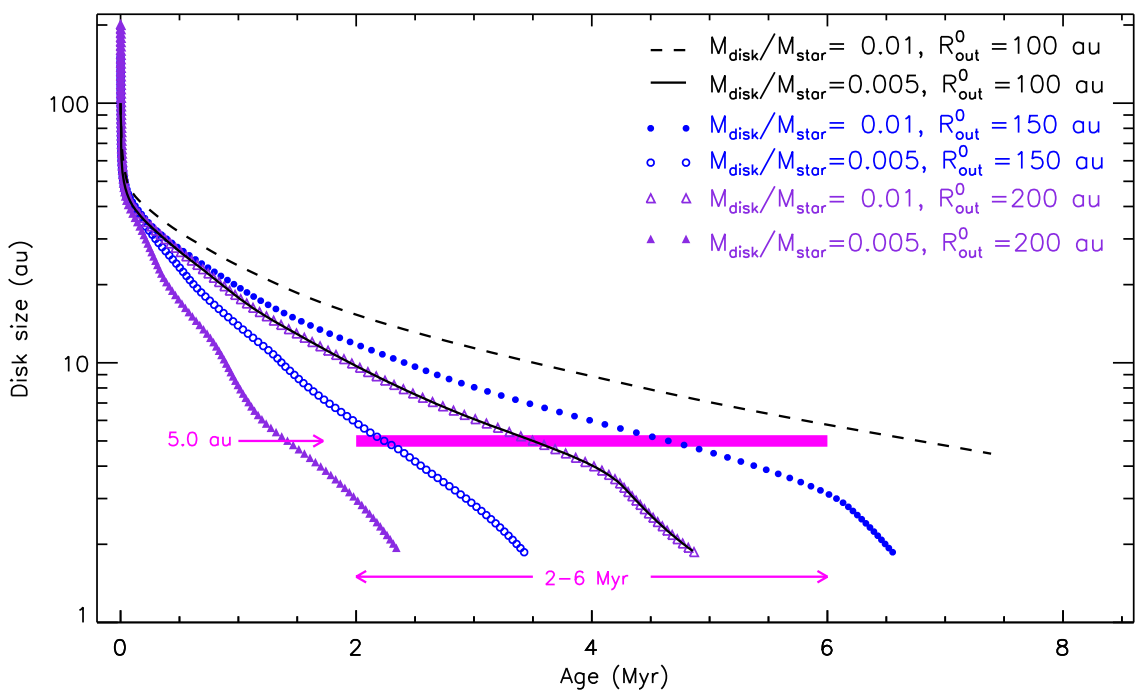

Figure 4. Sizes of disks around a $1.5 M_{\odot}$ young star decrease with age due to the external photoevaporation within the UV strength of $4470 G_{0}$, like KH $15 \mathrm{D}$. The different symbols are different starting disk masses and sizes.

strength $\left(4470 G_{0}\right)$, we make a toy model to assess the timescale for dissipating the outer disk of KH $15 \mathrm{D}$ as close in as $5 \mathrm{au}$. In the models, the initial disk sizes are set to be 100 , 150 , or $200 \mathrm{au}$, disk masses are 0.0075 or $0.015 M_{\odot}(0.5 \%$ or $1 \%$ of the $\mathrm{KH} 15 \mathrm{D}$ system mass), and the inner disk radius is fixed to be $0.1 \mathrm{au}$. The disk material has a surface density $\Sigma \propto r^{-1}$, and the central binary is simplified as one single star with a mass of $1.5 M_{\odot}$. Furthermore, we assume that the bulk of the mass loss due to external photoevaporation is driven from the disk outer edge, as in Haworth et al. (2018), and neglect the disk viscous evolution. We interpolate the disk mass-loss rates within the UV field strength of $4470 G_{0}$ during the disk dissipation using the FRIED grid of mass-loss rates for externally irradiated protoplanetary disks (see details in Haworth et al. 2018). The mass-loss rates range from $\sim 10^{-6}$ to $\sim 10^{-10} M_{\odot} \mathrm{yr}^{-1}$ when disk size decreases from hundreds au to $5 \mathrm{au}$. The resulting disk sizes from our toy models are shown in Figure 4 as a function of age. Our calculations show that the size of a disk within the UV field strength of $4470 G_{0}$ can decrease to $5 \mathrm{au}$ at the age of the cluster (2-6 Myr, Naylor 2009; Lim et al. 2016). Thus, photoevaporation can explain the outer truncation of the disk.

\section{Conclusion}

We provide a new interpretation to the double-peaked [O I] $\lambda 6300$ emission line as arising from the hot surface of the gaseous disk surrounding the spectroscopic binary KH 15D. Line profile modeling constrains the emission to a relatively narrow radial extent $\sim 0.5-5 \mathrm{au}$, similar to the extent of the dust ring inferred from modeling the source photometric variability. The relatively large inner edge is likely set by the binary dynamical interaction. We show that the small outer disk radius could be shaped by external photoevaporation driven by UV photons from the nearby massive star HD 47887. Newer, high $\mathrm{S} / \mathrm{N}$ [O I] $\lambda 6300$ line profiles would be useful to further test our interpretation and detect any subtle changes that might arise from disk precession.

We thank the anonymous referee for comments that helped to improve this Letter, and K. Kratter for inputs on the theory of circumbinary disks. I.P. and S.E. acknowledge support from a Collaborative NSF Astronomy \& Astrophysics Research Grant (ID: 1715022 and ID:1714229). This material is based upon work supported by the National Aeronautics and Space Administration under Agreement No. NNX15AD94G for the program "Earths in Other Solar Systems". The results reported herein benefitted from collaborations and/or information exchange within NASA Nexus for Exoplanet System Science (NExSS) research coordination network sponsored by NASA's Science Mission Directorate.

\section{Appendix \\ Comparison of Observed [O I] $\lambda 6300$ Line Profiles and the Model Line Profile}

Figure 5 shows the comparison of the line profiles observed at different epochs (gray and red lines) with the best-fit model line profile (blue lines). The latter is obtained by fitting the 2001 December 21 profile (outermost right panel). We chose this line profile because (1) it has the highest $\mathrm{S} / \mathrm{N}$, and (2) it shows the least contamination from sky lines. The best-fit parameters are: $\alpha=-2.82, r_{\text {in }}=0.54 \mathrm{au}$, and $r_{\text {out }}=5.0 \mathrm{au}$, see also Section 4. 


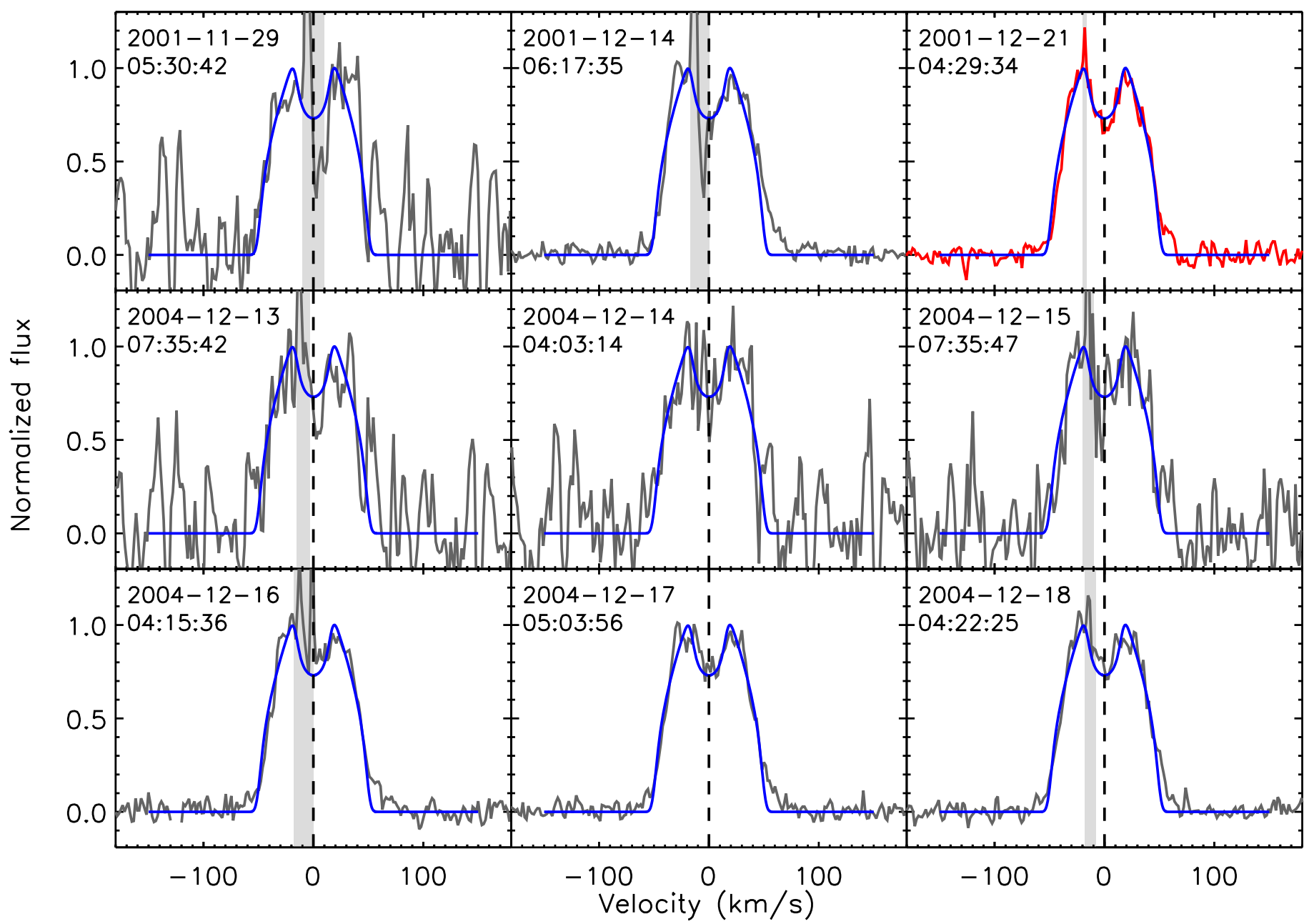

Figure 5. [O I] $\lambda 6300$ line profiles (gray and red lines) obtained between 2001 and 2004, compared to our best-fit line profile (blue lines), see the Appendix for details. The red line shows the [O I] $\lambda 6300$ line profile taken on 2001 December 21 and used for the fitting. For multiple observations during 2001 December 14 and 2004 December 13-16, we only show one spectrum per night as there are no detectable variations in [O I] $\lambda 6300$ profiles. The zero velocity marked with the dashed line is that of the KH 15D rest frame. The gray filled region marks wavelength ranges where sky contamination is likely.

\section{ORCID iDs}

Min Fang $\odot$ https://orcid.org/0000-0001-8060-1321 Ilaria Pascucci i https://orcid.org/0000-0001-7962-1683 Jinyoung Serena Kim (ㄱ) https://orcid.org/0000-0001-6072-9344

\section{References}

Ansdell, M., Williams, J. P., van der Marel, N., et al. 2016, ApJ, 828, 46 Aronow, R. A., Herbst, W., Hughes, A. M., Wilner, D. J., \& Winn, J. N. 2018, AJ, 155,47

Artymowicz, P., \& Lubow, S. H. 1994, ApJ, 421, 651

Arulanantham, N. A., Herbst, W., Cody, A. M., et al. 2016, AJ, 151, 90

Banzatti, A., Pascucci, I., Edwards, S., et al. 2019, ApJ, 870, 76

Capelo, H. L., Herbst, W., Leggett, S. K., Hamilton, C. M., \& Johnson, J. A. 2012, ApJL, 757, L18

Chiang, E. I., \& Murray-Clay, R. A. 2004, ApJ, 607, 913

Deming, D., Charbonneau, D., \& Harrington, J. 2004, ApJL, 601, L87

Fang, M., Pascucci, I., Edwards, S., et al. 2018, ApJ, 868, 28

Fossati, L., Zwintz, K., Castro, N., et al. 2014, A\&A, 562, A143

Freudling, W., Romaniello, M., Bramich, D. M., et al. 2013, A\&A, 559, A96

Hamilton, C. M., Herbst, W., Mundt, R., Bailer-Jones, C. A. L., \& Johns-Krull, C. M. 2003, ApJL, 591, L45

Hamilton, C. M., Herbst, W., Shih, C., \& Ferro, A. J. 2001, ApJL, 554, L201 Hamilton, C. M., Johns-Krull, C. M., Mundt, R., Herbst, W., \& Winn, J. N. 2012, ApJ, 751, 147

Hartigan, P., Edwards, S., \& Ghandour, L. 1995, ApJ, 452, 736

Hartigan, P., Frank, A., Foster, A. M., et al. 2011, ApJ, 736, 29

Haworth, T. J., Clarke, C. J., Rahman, W., Winter, A. J., \& Facchini, S. 2018, MNRAS, 481, 452
Herbst, W., Hamilton, C. M., Vrba, F. J., et al. 2002, PASP, 114, 1167 Johnson, J. A., Marcy, G. W., Hamilton, C. M., Herbst, W., \& Johns-Krull, C. M. 2004, AJ, 128, 1265

Kearns, K. E., \& Herbst, W. 1998, AJ, 116, 261

Kharchenko, N. V. 2001, KFNT, 17, 409

Kounkel, M., Hartmann, L., Tobin, J. J., et al. 2016, ApJ, 821, 8

Lanz, T., \& Hubeny, I. 2007, ApJS, 169, 83

Lim, B., Sung, H., Kim, J. S., et al. 2016, ApJ, 831, 116

Lin, D. N. C., \& Papaloizou, J. C. B. 1993, in Protostars and Planets III ed. E. H. Levy \& J. I. Lunine (Tucson, AZ: Univ. Arizona Press) 749

Lodato, G., \& Facchini, S. 2013, MNRAS, 433, 2157

Meijerink, R., Aresu, G., Kamp, I., et al. 2012, A\&A, 547, A68

Mundt, R., Hamilton, C. M., Herbst, W., Johns-Krull, C. M., \& Winn, J. N. 2010, ApJL, 708, L5

Muñoz, D. J., \& Lai, D. 2016, ApJ, 827, 43

Muñoz, D. J., Miranda, R., \& Lai, D. 2019, ApJ, 871, 84

Muzerolle, J., Flaherty, K., Balog, Z., et al. 2019, ApJ, 877, 29

Naylor, T. 2009, MNRAS, 399, 432

Pascucci, I., Testi, L., Herczeg, G. J., et al. 2016, ApJ, 831, 125

Pichardo, B., Sparke, L. S., \& Aguilar, L. A. 2008, MNRAS, 391, 815

Sharma, S. 2017, ARA\&A, 55, 213

Simon, M. N., Pascucci, I., Edwards, S., et al. 2016, ApJ, 831, 169

Thun, D., Kley, W., \& Picogna, G. 2017, A\&A, 604, A102

Tokunaga, A. T., Dahm, S., Gässler, W., et al. 2004, ApJL, 601, L91

Venuti, L., Prisinzano, L., Sacco, G. G., et al. 2018, A\&A, 609, A10

Windemuth, D., \& Herbst, W. 2014, AJ, 147, 9

Winn, J. N., Hamilton, C. M., Herbst, W. J., et al. 2006, ApJ, 644, 510

Winn, J. N., Holman, M. J., Johnson, J. A., Stanek, K. Z., \& Garnavich, P. M. 2004, ApJL, 603, L45 